\title{
Chromoendoscopy for Surveillance in Inflammatory Bowel Disease Does Not Increase Neoplasia Detection Compared With Conventional Colonoscopy With Random Biopsies: Results From a Large Retrospective
} Study

\author{
E. Mooiweer, MD, PhD ${ }^{1}$, A.E. van der Meulen-de Jong, MD, PhD², C.Y. Ponsioen, MD, PhD ${ }^{3}$, H.H. Fidder, MD, PhD ${ }^{1}$, \\ P.D. Siersema, MD, $\mathrm{PhD}^{1}$, E. Dekker, MD, $\mathrm{PhD}^{3}$ and B. Oldenburg, MD, $\mathrm{PhD}^{1}$
}

OBJECTIVES: $\quad$ Randomized trials demonstrated that chromoendoscopy is superior to white light endoscopy with random biopsy sampling (WLE) for the detection of dysplasia in patients with inflammatory bowel disease (IBD). Whether implementing chromoendoscopy can increase the detection of dysplasia in clinical practice is unknown.

METHODS: $\quad$ Patients with ulcerative colitis (UC) and Crohn's disease (CD) undergoing colonoscopic surveillance between January 2000 and November 2013 in three referral centers were identified using the patients' medical records. In recent years, the use of high-definition chromoendoscopy was adopted in all three centers using segmental pancolonic spraying of $0.1 \%$ methylene blue or $0.3 \%$ indigo carmine (chromoendoscopy group). Previously, surveillance was performed employing WLE with random biopsies every $10 \mathrm{~cm}$ (WLE group). The percentage of colonoscopies with dysplasia was compared between both groups.

RESULTS: $\quad$ A total of $\mathbf{4 4 0}$ colonoscopies in $\mathbf{4 0 1}$ patients were performed using chromoendoscopy and 1,802 colonoscopies in 772 patients using WLE. Except for a higher number of CD patients with extensive disease and more patients with a first-degree relative with colorectal cancer (CRC) in the chromoendoscopy group, the known risk factors for IBD-associated CRC were comparable between both groups. Dysplasia was detected during 48 surveillance procedures (11\%) in the chromoendoscopy group as compared with 189 procedures $(10 \%)$ in the WLE group $(P=0.80)$. Targeted biopsies yielded 59 dysplastic lesions in the chromoendoscopy group, comparable to the 211 dysplastic lesions detected in the WLE group ( $P=0.30)$.

CONCLUSIONS: Despite compelling evidence from randomized trials, implementation of chromoendoscopy for IBD surveillance did not increase dysplasia detection compared with WLE with targeted and random biopsies.

Am J Gastroenterol 2015; 110:1014-1021; doi:10.1038/ajg.2015.63; published online 31 March 2015

\section{INTRODUCTION}

Patients with longstanding ulcerative colitis (UC) and Crohn's disease $(\mathrm{CD})$ with colonic involvement have an increased risk of developing colorectal cancer (CRC) $(1,2)$. Endoscopic surveillance aimed at the detection and treatment of dysplasia and CRC at an early stage is advocated to mitigate this risk, although solid evidence that this strategy is effective is lacking (3). The detection of neoplasia is challenging, as lesions containing neoplasia are often flat or may not be endoscopically visible at all. Therefore, until recently, surveillance guidelines recommended taking

${ }^{1}$ Department of Gastroenterology and Hepatology, University Medical Center Utrecht, Utrecht, The Netherlands; ${ }^{2}$ Department of Gastroenterology and Hepatology, Leiden University Medical Center, Leiden, The Netherlands; ${ }^{3}$ Department of Gastroenterology and Hepatology, Amsterdam Medical Center, Amsterdam, The Netherlands. Correspondence: Bas Oldenburg, MD, PhD, Department of Gastroenterology and Hepatology, University Medical Center Utrecht, Heidelberglaan 100, 3584 CX Utrecht, The Netherlands. E-mail: b.oldenburg@umcutrecht.nl

Received 14 August 2014; accepted 3 February 2015 
multiple random biopsies throughout the entire colon, although 40 to 50 biopsies are needed to achieve an acceptable accuracy for detecting neoplasia $(4,5)$. Moreover, the neoplasia yield of these random biopsies is disappointingly low (6). In addition, recently published studies show that almost all neoplastic lesions can be identified endoscopically nowadays, casting further doubt on the practice of taking multiple random biopsies for surveillance purposes (6-8).

Several randomized trials reported that chromoendoscopy using indigo carmine or methylene blue can increase the neoplasia detection rate substantially compared with white light endoscopy with random biopsy sampling (WLE) (9-12). These findings have prompted the British Society of Gastroenterology and American Gastroenterological Association to advocate chromoendoscopy as the method of choice for CRC surveillance in their updated guidelines $(13,14)$. Whether the broad implementation of chromoendoscopy in clinical practice indeed increases the neoplasia detection rate compared with WLE is currently unknown. The aim of this study was therefore to compare the neoplasia detection rate of colonoscopies performed using chromoendoscopy with procedures performed with WLE.

\section{METHODS}

\section{Patients}

Patients with a diagnosis of CD or UC were identified in three referral centers using the diagnosis treatment combinations for inflammatory bowel disease (IBD). Diagnosis treatment combinations are based on the International Classification of Disease, 9th Revision, and can be considered the Dutch version of the diagnosis-related groups that are used in other countries.

The medical records and endoscopy reports were reviewed to establish whether patients had a valid indication for CRC surveillance, that is, patients with a disease duration of $\geq 8$ years and cumulative colonic involvement of at least left-sided colitis (UC and IBD-unclassified patients) or $>30 \%$ of the colonic mucosa (CD patients). In addition, patients with colitis and primary sclerosing cholangitis were eligible as soon as the combination of these diagnoses was established.

The medical records were also reviewed to obtain the patients' demographics, date of IBD diagnosis, type of IBD, disease extent before the start of surveillance, and family history of CRC.

Details about the family history of CRC were obtained from a questionnaire as part of an observational cohort study for the subgroup of patients who underwent chromoendoscopy.

\section{Surveillance colonoscopies}

All surveillance colonoscopies performed between January 2000 and November 2013 in patients with a valid indication for surveillance were collected. Colonoscopies were classified as a surveillance procedure when this was explicitly stated as the indication for the colonoscopy and when either random biopsies were taken or chromoendoscopy was performed.

At the start of the study period, the three centers performed surveillance employing WLE with targeted biopsies of suspicious lesions in combination with four quadrant random biopsies every
$10 \mathrm{~cm}$ throughout the entire colon, in accordance with the international guidelines (WLE group) $(4,5)$. In recent years, all centers adopted pancolonic chromoendoscopy with spraying of either $0.3 \%$ indigo carmine or $0.1 \%$ methylene blue and targeted biopsies of suspicious lesions as the preferred surveillance method (chromoendoscopy group). Dye was applied using a spray catheter (Olympus PW-205V, Olympus Europe, Hamburg, Germany) one colonic segment at a time during extubation. Upon reintroduction, excess dye was suctioned of and during the second withdrawal the colonic mucosa was inspected.

Endoscopists received no specific training for the technique of chromoendoscopy before its implementation, although each surveillance procedure during the study period was performed by or under close supervision of gastroenterologists with extensive experience in dysplasia surveillance in IBD. Each surveillance colonoscopy was included in the chromoendoscopy or the WLE group based on the method used as described in the endoscopy report. As this was a retrospective analysis over a 13-year period, multiple surveillance colonoscopies per patient were performed and therefore patients could be included in the WLE group as well as in the chromoendoscopy group.

Procedures in which bowel preparation was deemed inadequate by the endoscopist or in which the cecum was not reached were excluded. Surveillance procedures that were aborted because of the presence of severe inflammation were excluded as well. The participating centers employed the British Society of Gastroenterology guidelines published in 2002 to schedule the next surveillance procedure throughout the study period (5).

\section{Neoplasia}

The size, location, and endoscopic description of all lesions suspected of containing neoplasia that were biopsied or removed endoscopically or surgically were recorded. Lesions were classified as nonneoplastic, low-grade dysplasia (LGD), high-grade dysplasia, or CRC based on the pathology report. Discrete solitary sessile or pedunculated polyps resembling sporadic adenomas and showing adenomatous tissue on histology were classified as adenomas, whereas all other endoscopic abnormalities (i.e., plaque-like lesions, irregular masses) containing neoplasia were classified as nonadenoma-like masses. In the WLE group, both the total number of random biopsies and the presence of neoplasia in these biopsies were recorded.

\section{Comparison between chromoendoscopy and WLE procedures}

The percentage of colonoscopies with neoplasia (neoplasia yield) was compared between all procedures performed with chromoendoscopy and those performed with WLE. In case of the WLE group, this comprised neoplasia in targeted as well as random biopsy samples. The total number of endoscopically visible lesions containing neoplasia was also compared between both groups.

In the subgroup of patients in whom a WLE procedure was followed by a chromoendoscopy procedure during the study period, a direct comparison of the neoplasia detection rate between both surveillance methods was made within the same patient. As multiple consecutive surveillance procedures might decrease the 
neoplasia yield of later colonoscopies, the same comparison was made between all patients in whom two subsequent WLE procedures were performed as a reference.

\section{Statistics}

Baseline characteristics of the chromoendoscopy and WLE groups were compared using Pearson's $\chi^{2}$ analysis for categorical variables and Student's $t$-test or Mann-Whitney $U$-test for continuous variables, depending on whether data were normally distributed. The percentage of colonoscopies with neoplasia was compared between the chromoendoscopy and WLE groups using Pearson's $\chi^{2}$ analysis.

The comparison between the neoplasia detection rate of chromoendoscopy and WLE within the same patients was made using the McNemar test. A $P$ value of $<0.05$ was considered statistically significant. Statistical analysis was performed using SPSS version 20 (Chicago, IL) for Windows.

\section{RESULTS}

In total, 937 patients (35\% CD and 65\% UC or IBD-unclassified) underwent 2,242 surveillance colonoscopies during the study period. Chromoendoscopy was performed in 440 procedures in 401 patients, whereas WLE was performed in 1,802 procedures in 772 patients (Figure 1). The percentage of patients excluded because of inadequate bowel preparation was similar between the WLE and chromoendoscopy groups (15\% vs. $12 \%$ respectively, $P=0.25)$. Baseline characteristics are shown in Table 1. A subgroup of 236 patients underwent WLE as well as chromoendoscopy during the study period. The number of $\mathrm{CD}$ patients with extensive colitis and the number of patients with a first-degree relative diagnosed with CRC were significantly higher in the chromoendoscopy group (66\% vs. $51 \%$ and $16 \%$ vs. $4 \%$, both $P<0.01$, Table 1). The difference in the percentage of patients with a positive family history of CRC could be explained by missing data in the WLE group, as the difference was no longer significant after excluding patients with missing data ( $22 \%$ vs. $24 \%, P=0.72$ ). Furthermore, the mean interval between the prior "pre-study" surveillance colonoscopy and the chromoendoscopy procedure was significantly longer compared with the surveillance interval in the WLE group (2.8 compared with 2.4 years, $P=0.01$ ). There were no significant differences between the chromoendoscopy and WLE group with regard to other established risk factors for IBD-associated CRC (Table 1).

\section{Neoplasia detection rate}

In total, neoplasia was detected in 237 surveillance colonoscopies (11\%) during the study period. LGD was detected in 227 procedures $(10 \%)$, high-grade dysplasia in 6 procedures $(0.3 \%)$, and CRC in 4 procedures $(0.2 \%)$. The overall neoplasia detection rate was comparable between the three centers (11\% vs. $9 \%$ vs. $11 \%$ respectively, $P=0.45$ ). When surveillance colonoscopies were reviewed in chronological order, the neoplasia detection rate remained stable over time (Figure 2). The number of surveillance procedures that each patient underwent had no effect on the neoplasia detection rate. In $9 \%$ of cases, dysplasia was detected during the first procedure that was comparable to the detection rate in the second (9\%), third (15\%), and fourth (10\%) surveillance colonoscopies among the patients who underwent multiple consecutive surveillance procedures $(P=0.10$, Figure 3$)$.

\section{Neoplasia detection per surveillance method}

Neoplasia was detected in 48 out of 440 surveillance procedures performed with chromoendoscopy ( $11 \%, 95 \%$ confidence interval (CI) $8-14 \%$ ) and in 189 of the 1,802 procedures performed with WLE $(10 \%, 95 \%$ CI $9-12 \%, P=0.80)$. The cumulative neoplasia detection rate over time for each surveillance technique is shown in Figure 4. If the 236 patients who underwent both chromoendoscopy and WLE were excluded from the analysis, the neoplasia detection rate remained comparable between the chromoendoscopy group (10\%, 95\% CI 6-14\%) and the WLE group (11\%, 95\% CI 9-13\% $P=0.65)$. The similarity in neoplasia detection between chromoendoscopy and WLE procedures was observed in all three centers ( $11 \%$ vs. $11 \%, P=0.91,8 \%$ vs. $9 \%, P=0.65,12 \%$ vs. $10 \%$, $P=0.60$ respectively).

\section{Comparison between chromoendoscopy and WLE procedures within the same patients}

Of the 236 patients who underwent both chromoendoscopy and WLE during the study period, 5 were excluded because they underwent WLE after chromoendoscopy. Neoplasia was detected in $31(13 \%, 95 \%$ CI 9-18\%) of the WLE procedures and in $30(13 \%, 95 \%$ CI $9-18 \%)$ of the chromoendoscopy procedures $(P=1.0)$. As a reference, 661 paired consecutive WLE procedures were available, of which the neoplasia detection rate was $8 \%(95 \%$ CI 6-10\%) in the first procedure compared with $11 \%$ (95\% CI $9-13 \%)$ in the following procedure $(P=0.05)$.

\section{Neoplasia characteristics per surveillance method}

In the chromoendoscopy group, targeted biopsies were sampled from 546 lesions (mean 1.2 per procedure) that was significantly higher than the 1,069 lesions (mean 0.6 per procedure) biopsied in the WLE group $(P<0.01$, Table 2$)$. The targeted biopsies in the chromoendoscopy group identified neoplasia in 48 procedures $(11 \%)$, similar to the 158 procedures $(9 \%)$ with neoplasia identified with targeted biopsies in the WLE group $(P=0.19)$. The number of lesions with neoplasia was also comparable between the chromoendoscopy and WLE groups ( $P=0.30$, Table 2$)$.

The 51,602 random biopsies sampled in the WLE group yielded an additional 31 procedures (1\%) in which neoplasia was detected. In all cases, this was found to be LGD.

\section{Learning curve}

Two endoscopists performed at least 50 consecutive surveillance colonoscopies with chromoendoscopy allowing analysis of a potential learning curve for this technique. The neoplasia detection rate when procedures are placed in chronological order for both endoscopists is shown in Figure 5. The neoplasia detection rate increased from 5 to $16 \%$ for one endoscopist $(P=0.14)$, but decreased from 17 to $8 \%$ for the second endoscopist $(P=0.09)$ 


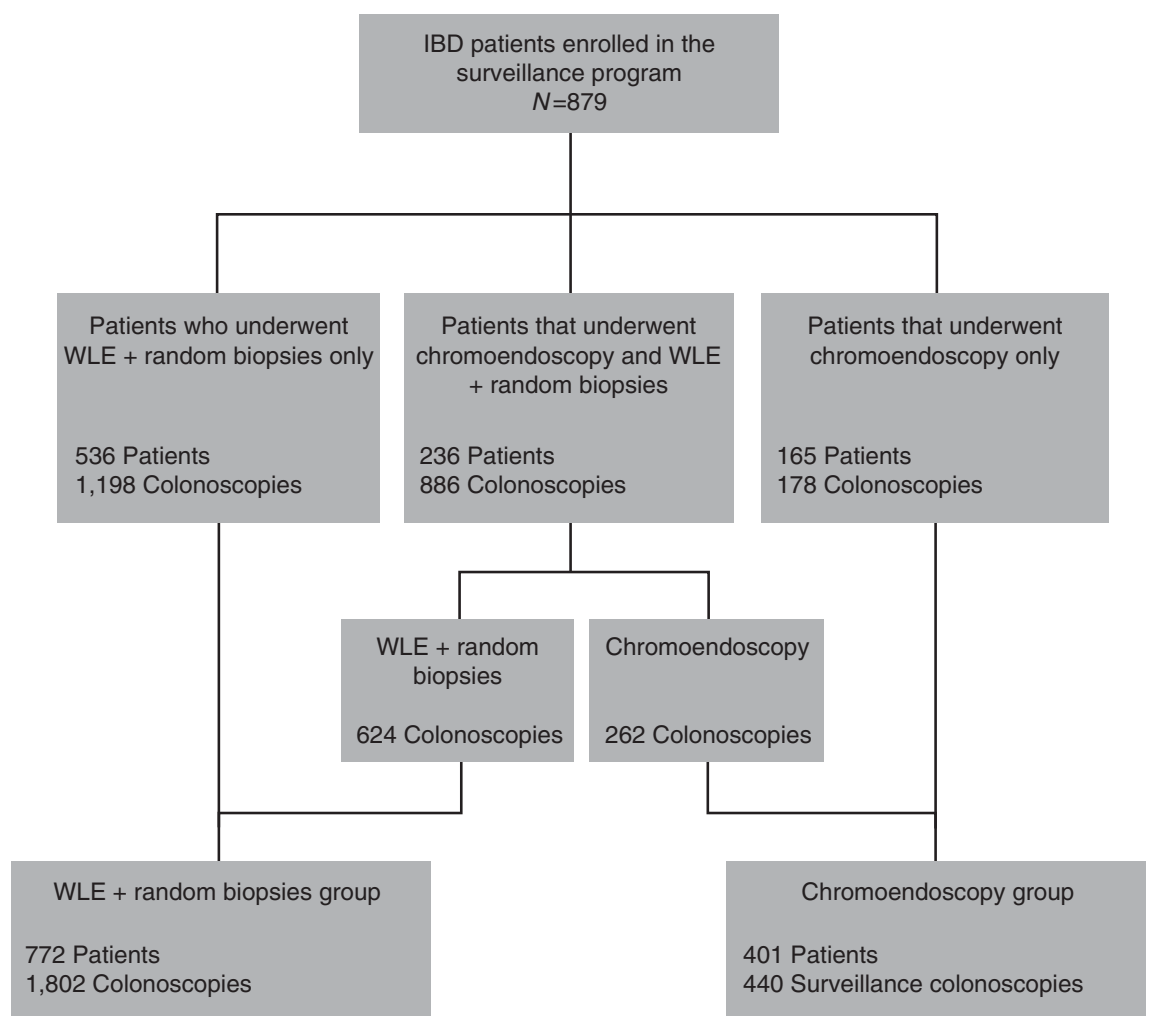

Figure 1. Flowchart showing the patients included in each group. IBD, inflammatory bowel disease; WLE, white light endoscopy with random biopsy sampling.

when the first $50 \%$ of procedures were compared with the second $50 \%$ of procedures.

\section{DISCUSSION}

This large retrospective study showed that the implementation of chromoendoscopy with targeted biopsies as the preferred surveillance method did not result in an increased neoplasia detection rate as compared with WLE with random biopsies.

This finding is in contrast with several controlled trials that showed a substantial increase in neoplasia detection using chromoendoscopy compared with WLE $(9-12)$. Kiesslich et al. $(10,12)$ reported an impressive increase of dysplasia yield using chromoendoscopy as compared with WLE from 6 to 15\% (combined data from 2 randomized controlled trials). Two other studies employing a back-to-back study design reported that among 202 procedures, the second withdrawal using chromoendoscopy increased the neoplasia detection rate from 7 to $13 \%(9,11)$. As dye spraying cannot be undone, the two back-to-back studies could not perform WLE after chromoendoscopy, and this may have overestimated the additional neoplasia yield of chromoendoscopy in these particular studies. Studies in non-IBD patients using a back-to-back design have shown that the second colonoscopy, even when using standard WLE colonoscopy in both cases, increases the adenoma detection rate substantially (15). It is therefore conceivable that the same phenomenon could have resulted in higher yields in the second procedure in these colitis studies as well. Furthermore, the endoscopist cannot be blinded for the surveillance method. It could be that the expectation that chromoendoscopy performs better introduced a bias, despite randomization.

The combined neoplasia detection rate using WLE from the randomized trials and back-to-back studies was substantially lower than the rate in our study ( $7 \%$ vs. $10 \%)$, whereas the rate for chromoendoscopy was found to be higher compared with our results ( $14 \%$ vs. $11 \%)$. These divergent outcomes may be because of several factors. First, the higher neoplasia detection rates in previous trials might be because of the fact that expert endoscopists with extensive experience performed the chromoendoscopy procedures. Obviously, this would not explain the lower neoplasia yield of the WLE arm in the randomized controlled trials. As all three centers in our study started using chromoendoscopy without a training period before its implementation, the initial learning curve might have lowered the total neoplasia detection rate, especially compared with the experts who performed chromoendoscopy in previous randomized trials. We investigated whether a learning curve was present in two endoscopists and found no significant increase in neoplasia detection over time, suggesting there was no substantial learning curve. Of note, the procedures in our cohort were performed or supervised by endoscopists with extensive experience in surveillance colonoscopies for colitis, providing a near optimal setting for surveillance. Second, the absence of a strict protocol for surveillance colonoscopies in the three participating centers may 
Table 1. Baseline characteristics of the patients who underwent chromoendoscopy and WLE+random biopsies

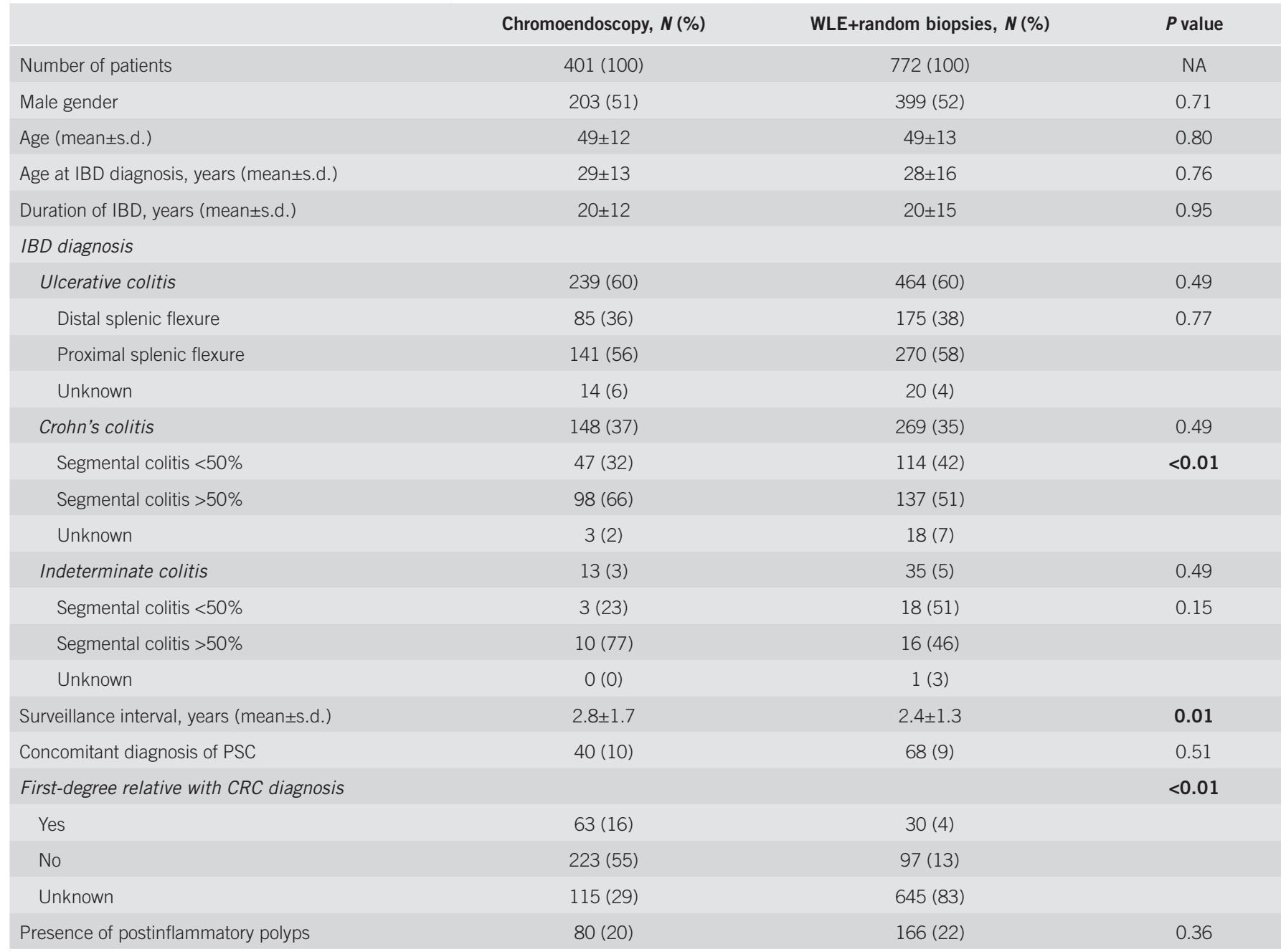

CRC, colorectal cancer; IBD, inflammatory bowel disease; NA, not applicable; PSC, primary sclerosing cholangitis; WLE, white light endoscopy with random biopsy sampling. $P$ values shown in bold reflect a significant difference between the two groups ( $\chi^{2}$ test).

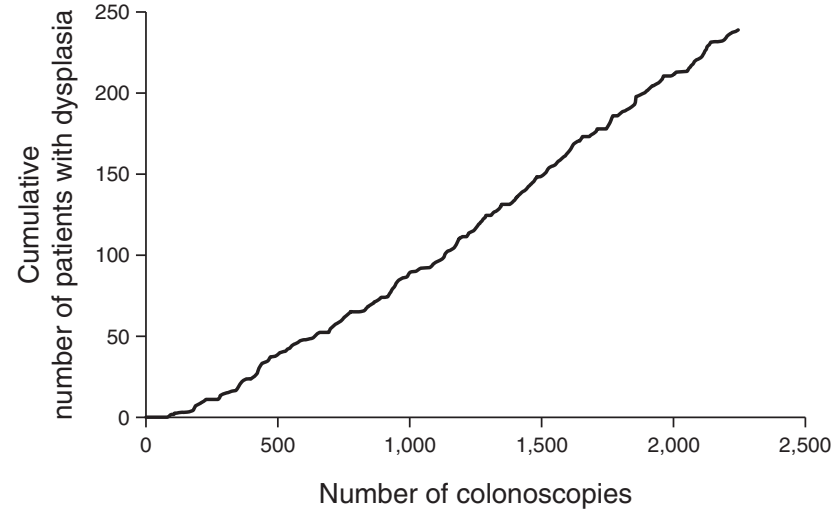

Figure 2. Neoplasia detection rate over time when each surveillance colonoscopy is placed in chronological order.

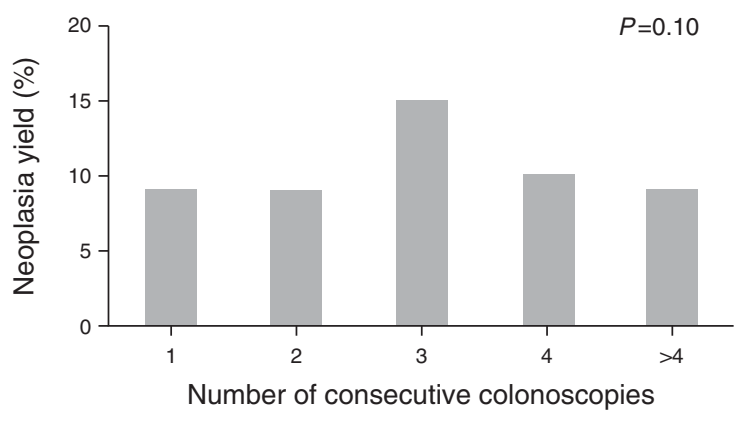

$\begin{array}{lllll}\text { Number of patients } & 937 & 582 & 360 & 191\end{array}$

Figure 3. Neoplasia detection rate among patients who underwent multiple surveillance colonoscopies during the study period. WLE, white light endoscopy with random biopsy sampling. 
have affected the quality of the procedures. As bowel preparation and the absence of inflammation are paramount for the detection of subtle dysplastic lesions, especially when using chromoendoscopy (16), the lack of a standardized approach might have resulted in the inclusion of low-quality procedures and missed lesions in our study. Again, this would not explain the relative low neoplasia yield in the randomized controlled trials and high neoplasia yield in our series in the WLE group. Third, as the study period spanned more than a decade, different types of endoscopes were used over time, and this could have influenced neoplasia detection rates. Although data on the type and characteristics of the endoscopes used were not collected, the bulk of the chromoendoscopy procedures was performed in the past few years of the study period and therefore these procedures benefitted from better endoscopes

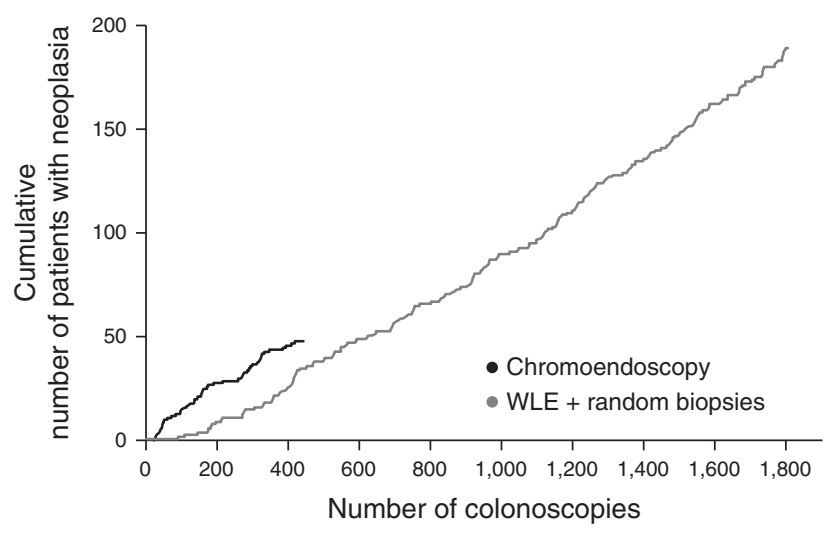

Figure 4. Neoplasia detection rate over time for chromoendoscopy and white light endoscopy with random biopsy sampling (WLE). with a higher resolution. This could have led to a higher neoplasia detection rate in our chromoendoscopy group and a gradual overall increase of neoplasia yield in both the chromoendoscopy and WLE groups over time. However, the data from the current study do not support this assumption (Figure 2).

The retrospective nature of our study is an important limitation, and this means our results should be interpreted with caution as this might have introduced several forms of bias. As our study covers a period of $>10$ years, it is conceivable that a change of the incidence of neoplasia has influenced our results. Several epidemiological studies have shown that the risk of CRC is decreasing in IBD patients, possibly because of improved treatment and/or better implementation of endoscopic surveillance $(17,18)$. By plotting each surveillance colonoscopy in chronological order,

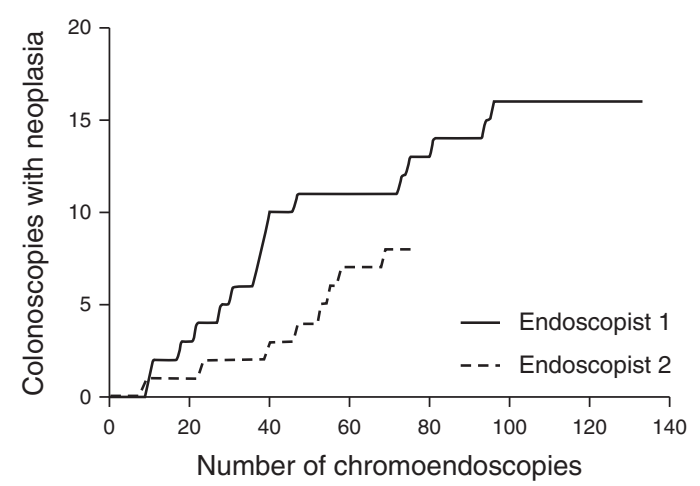

Figure 5. Neoplasia detection rate over time for chromoendoscopies performed consecutively by two endoscopists to assess the presence of a learning curve.

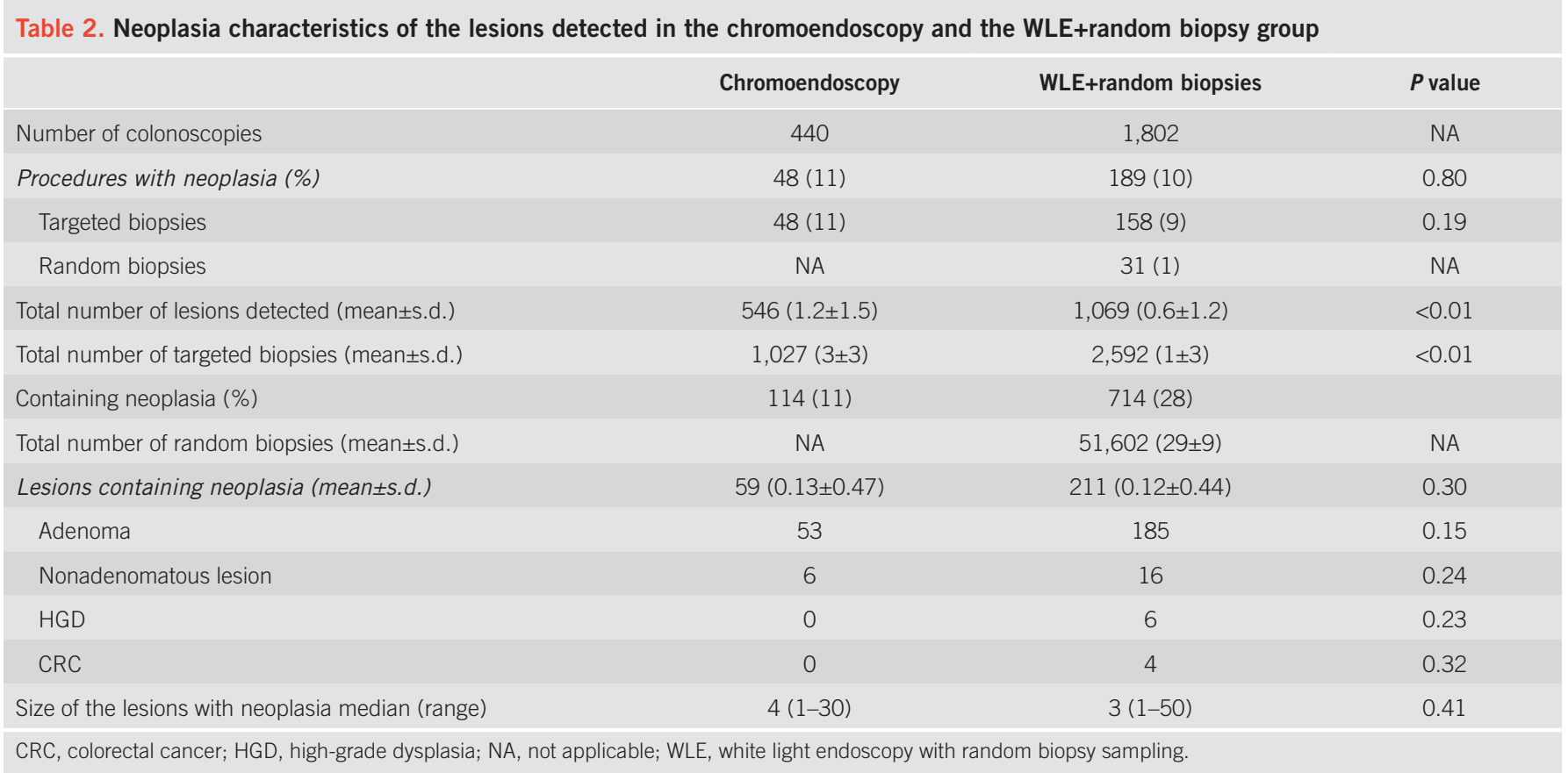


we found that the neoplasia detection rate remained remarkably stable throughout the study period, suggesting this did not influence our results (Figure 2). Theoretically, however, the decreased incidence of neoplasia in IBD over time could have been balanced out by the increased neoplasia yield using chromoendoscopy and/ or better endoscopes.

The fact that this study was retrospective and not randomized might well have resulted in a difference in patient characteristics between the chromoendoscopy and WLE groups. We indeed found that the percentage of $\mathrm{CD}$ patients with extensive colitis and patients with a positive family history for CRC was higher in the chromoendoscopy group. As these are both risk factors for CRC, these differences should have caused a higher rather than a lower dysplasia detection rate. The interval between the last "pre-study" surveillance colonoscopy and the procedures performed with chromoendoscopy was also significantly longer compared with the surveillance intervals in the WLE group. Again, as a longer surveillance interval theoretically results in more time for neoplasia to develop, this bias would only increase the neoplasia detection rate. Of course, some level of residual confounding is undoubtedly present.

Despite the fact that there were no significant differences in both the number of lesions or procedures with neoplasia between the chromoendoscopy and WLE groups, it is conceivable that chromoendoscopy aids in the detection of more subtle, small, and flat dysplastic lesions (10). The fact that the size of the dysplastic lesions was comparable in both groups does not support this notion however.

Another issue that cannot be addressed by the current study design is the clinical relevance of the neoplastic lesions detected by both surveillance techniques. The ultimate goal of surveillance is to detect and treat dysplastic lesions that would otherwise progress to CRC and thereby decrease CRC-related mortality. Especially for LGD detected in random biopsies, there has been much debate on whether this finding is clinically relevant. Although some studies showed that between 37 and $53 \%$ of patients with confirmed LGD detected in random biopsies progress to high-grade dysplasia or CRC during follow-up $(19,20)$, others have reported that the incidence of dysplasia in random biopsies is extremely low and that this usually has no consequences for the follow-up strategy (6). The same holds true for the additional neoplasia detected with chromoendoscopy compared with conventional WLE. It has been suggested that these lesions are often small and flat, but there are no follow-up studies on the progression rates to advanced neoplasia of these lesions.

If future prospective studies will confirm our results, the question arises of what the role of chromoendoscopy should be in the setting of CRC surveillance in IBD patients. Chromoendoscopy was initially commended for its superior neoplasia detection, but it may also be less costly than WLE plus random biopsies as well that in itself could be an incentive to prefer chromoendoscopy (21). Furthermore, chromoendoscopy might aid in assessing the pit pattern of lesions and thereby aiding the differentiation between neoplastic and nonneoplastic lesions. On the other hand, it is conceivable that the targeted biopsies guided by the present, state-of-the-art, high-definition colonoscopes can provide the same neoplasia detection rates as chromoendoscopy without taking additional random biopsies, rendering chromoendoscopy as a flagging tool redundant in the near future.

In conclusion, we did not find an increase in neoplasia detection after the implementation of chromoendoscopy as compared with the conventional WLE plus random biopsies protocol. Although more studies are needed to confirm this and our results could be biased because of the retrospective nature of our study, these results cast doubt on the standard use of chromoendoscopy as the preferred surveillance tool in IBD.

\section{CONFLICT OF INTEREST}

Guarantor of the article: E. Mooiweer, $\mathrm{MD}, \mathrm{PhD}$.

Specific author contributions: E.M.: conceived the study, carried out the data collection and data analyses, and drafted the manuscript; A.E.M., C.Y.P., H.H.F., P.D.S., and E.D.: interpretation of data and helped draft the manuscript. B.O.: helped draft the manuscript, interpretation of the data, and participated in the design of the study. All authors read and approved the final manuscript before submission. Financial support: This project was supported by an unrestricted grant from MSD BV and an unrestricted grant from Ferring BV. Both had no influence in the study design, in the collection, analysis and interpretation of data; in the writing of the manuscript; and in the decision to submit the manuscript for publication.

Potential competing interests: None.

\section{Study Highlights}

\section{WHAT IS CURRENT KNOWLEDGE}

The risk of colorectal cancer in ulcerative colitis and Crohn's colitis is increased and therefore guidelines recommend colonoscopic surveillance in these patients.

$\checkmark$ Previously, surveillance was performed with white light endoscopy combined with the sampling of four random biopsies every $10 \mathrm{~cm}$ throughout the colon.

Pancolonic spraying of the colonic mucosa with indigo carmine or methylene blue (chromoendoscopy) has been found to significantly increase the dysplasia detection rate in several controlled trials.

\section{WHAT IS NEW HERE}

Implementing chromoendoscopy as the preferred surveillance method in recent years did not improve dysplasia detection rates as compared with white light endoscopy in a large "real-life" cohort.

Although this is a retrospective, nonrandomized study, these results cast doubt on the use of chromoendoscopy as the preferred surveillance technique in clinical practice. 
4. Winawer S, Fletcher R, Rex D et al. Colorectal cancer screening and surveillance: clinical guidelines and rationale-update based on new evidence. Gastroenterology 2003;124:544-60.

5. Eaden JA, Mayberry JF. Guidelines for screening and surveillance of asymptomatic colorectal cancer in patients with inflammatory bowel disease. Gut 2002;51:V10-2.

6. van den Broek FJ, Stokkers PC, Reitsma JB et al. Random biopsies taken during colonoscopic surveillance of patients with longstanding ulcerative colitis: low yield and absence of clinical consequences. Am J Gastroenterol 2011;109:715-22.

7. Rutter MD, Saunders BP, Wilkinson $\mathrm{KH}$ et al. Most dysplasia in ulcerative colitis is visible at colonoscopy. Gastrointest Endosc 2004;60:334-9.

8. Rubin DT, Rothe JA, Hetzel JT et al. Are dysplasia and colorectal cancer endoscopically visible in patients with ulcerative colitis? Gastrointest Endosc 2007;65:998-1004.

9. Rutter MD, Saunders BP, Schofield G et al. Pancolonic indigo carmine dye spraying for the detection of dysplasia in ulcerative colitis. Gut 2004;53:256-60.

10. Kiesslich R, Fritsch J, Holtmann M et al. Methylene blue-aided chromoendoscopy for the detection of intraepithelial neoplasia and colon cancer in ulcerative colitis. Gastroenterology 2003;124:880-8.

11. Marion JF, Waye JD, Present DH et al. Chromoendoscopy-targeted biopsies are superior to standard colonoscopic surveillance for detecting dysplasia in inflammatory bowel disease patients: a prospective endoscopic trial. Am J Gastroenterol 2008;103:2342-9.

12. Kiesslich R, Goetz M, Lammersdorf K et al. Chromoscopy-guided endomicroscopy increases the diagnostic yield of intraepithelial neoplasia in ulcerative colitis. Gastroenterology 2007;132:874-82.
13. Cairns SR, Scholefield JH, Steele RJ et al. Guidelines for colorectal cancer screening and surveillance in moderate and high risk groups (update from 2002). Gut 2010;59:666-89.

14. Farraye FA, Odze RD, Eaden J et al. AGA medical position statement on the diagnosis and management of colorectal neoplasia in inflammatory bowel disease. Gastroenterology 2010;138:738-45.

15. Rex DK, Cutler CS, Lemmel GT et al. Colonoscopic miss rates of adenomas determined by back-to-back colonoscopies. Gastroenterology 1997;112:24-8.

16. Kiesslich R, Neurath MF. Surveillance colonoscopy in ulcerative colitis: magnifying chromoendoscopy in the spotlight. Gut 2004;53:165-7.

17. Lutgens MW, van Oijen MG, van der Heijden GJ et al. Declining risk of colorectal cancer in inflammatory bowel disease: an updated metaanalysis of population-based cohort studies. Inflamm Bowel Dis 2013;19: 789-99.

18. Beaugerie L, Svrcek M, Seksik P et al. Risk of colorectal high-grade dysplasia and cancer in a prospective observational cohort of patients with inflammatory bowel disease. Gastroenterology 2013;145:166-75.

19. van Schaik FD, ten Kate FJ, Offerhaus GJ et al. Misclassification of dysplasia in patients with inflammatory bowel disease: consequences for progression rates to advanced neoplasia. Inflamm Bowel Dis 2011;17:1108-16.

20. Ullman T, Croog V, Harpaz N et al. Progression of flat low-grade dysplasia to advanced neoplasia in patients with ulcerative colitis. Gastroenterology 2003;125:1311-9.

21. Konijeti GG, Shrime MG, Ananthakrishnan AN et al. Cost-effectiveness analysis of chromoendoscopy for colorectal cancer surveillance in patients with ulcerative colitis. Gastrointest Endosc 2013;79:455-65. 